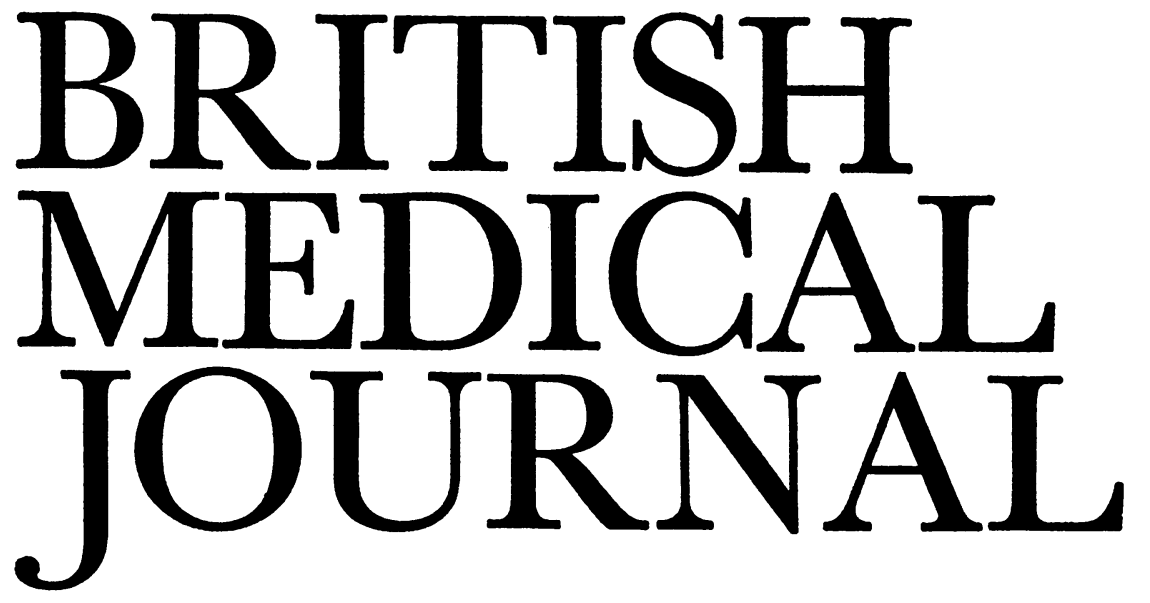

\title{
Short Boys
}

Concern about the slow growth of their child is a common reason for parents to seek medical advice. Anxiety is often greater when the child is a boy, and most doctors who deal with children are familiar with the problem of the schoolboy who is small for his age. While mixing with younger children in primary school he may not look or feel out of place, and difficulties may arise only when his secondary education begins and he is surrounded by bigger and more mature children. A youthful appearance with delayed pubertal development may add to his embarrassment and lead to much unhappiness.

Endocrine disorders are commonly suspected but rarely found in such patients. But in a few cases a history of normal early development followed by a fall in growth rate alerts the clinician to the presence of endocrine disease such as hypothyroidism or craniopharyngioma causing pituitary insufficiency. A chubby appearance associated with very small genitalia may provide a clue to long-standing growth hormone (HGH) deficiency, and delayed growth with progressive obesity may raise the possibility of Cushing's syndrome. In these situations further investigation is usually incicated, and laboratory methods for confirming the presence of such endocrine disorders are becoming more widely available. Medical conditions which interfere with growth, such as severe asthma or heart disease, are usually easily recognized, but some disorders may be more difficult to diagnose. In particular, there may be few clues to the presence of long-standing renal or gastrointestinal disease, and earlier symptoms such as abdominal discomfort, diarrhoea, or dysuria are often overlooked. Exclusion of mild coeliac disease by screening tests can be particularly difficult.

Occasionally examination will disclose other anomalies indicating that the patient may have one of the many clinical syndromes associated with abnoraml growth. ${ }^{1}$ Asymmetry of the limbs associated with low birthweight (Silver's syndrome) is probably one of the commoner syndromes of short stature, and its recognition may spare the patient unnecessary investigation. More commonly short stature following from low birth weight is not associated with other anomalies and is probably a sequel to reduced cell multiplication in utero.

In the great majority of patients no abnormality can be found to account for delayed growth. In many cases slow growth is clearly genetically determined and there is a strong family history of short stature. In others a family history of delayed pubertal development may be obtained. These patients very commonly show delayed bone age, which is usually assessed from radiographs of the wrist and hand. This finding implies that the growth period will be prolonged and that the patient has a chance to catch up with his peers in his late 'teens. Tables are available for predioting adult height for a given bone age, ${ }^{2}$ but as estimates of bone maturation may vary considerably between different observers ${ }^{3}$ errors may arise in such prediction.

Active treatment is not required for the majority of boys with slow growth and delayed puberty. Reassurance that there is no underlying disease, together with an explanation of what the future growth pattern is likely to be, usually allays anxieties. If doubts persist, measurement of height at intervals of 6 or 12 months almost invariably shows that the growth rate is normal and reassures the patient that he is not falling further and further behind his contemporaries. In a few patients, particularly those with predicted shortness or severe emotional difficulties, the question of hormone treatment is likely to be raised. It is generally accepted that HGH therapy is of no help to children with normal secretion of growth hormone, and at present androgen treatment is the only method available for speeding up growth. But the long-term value of this treatment is open to controversy. While numerous studies have shown that treatment with testosterone accelerates growth in a high proportion of patients, in some cases bone maturation has advanced more rapidly than height and so adult height may have been jeopardized. 45 Treatment with anabolic steroids carries a similar risk, as analogues of testosterone also accelerate bone maturation. 67 This hazard of disproportionately rapid bone maturation appears to be increased if high doses of androgen are given ${ }^{4}$ but is probably not particularly great when low doses are given for limited periods. J. G. Kaplan and his colleagues $^{8}$ have recently analysed the long-term results in 19 patients who had been treated for slow growth with modest doses of androgen. The majority of the patients reached or exceeded the height predicted before treatment. Twenty-one untreated patients were available as controls. Though fewer of these patients reached their predicted height, the difference between the two groups was within the bounds of chance, and there was no clear evidence that trearment had improved adult height.

These findings are very similar to those described by $\mathrm{N}$. Bayley and her colleagues 9 and by G. L. Foss ${ }^{10}$ in boys who 
had been treated for short stature with testosterone. In both series the majority of patients reached their predicted height and fared slightly better than untreated patients. However, patients with anorchia who were treated intensively with testosterone did less well and on average appeared to have lost about 3 in $(8 \mathrm{~cm})$ potential growth. ${ }^{10} \mathrm{R}$. Greene and L. S. Carstairs $^{11}$ also found that androgen treatment did not appear to reduce adult height. On the contrary, the final heights of 27 men who had been treated as children with testosterone exceeded the predicted heights by up to 9 in $(23 \mathrm{~cm})$. The significance of this finding is uncertain because a control group of untreated patients was not available, and the apparent benefit of treatment may merely reflect a general underestimation of predicted height.

Androgen therapy carries other potential risks, particularly when high doses are used. In theory the secretion of gonadotrophin may be suppressed, but there is no evidence that this is a serious long-term hazard. 9 More important, gynaecomastia, jaundice, and abnormal liver function tests have been reported, and treatment of aplastic anaemia with anabolic steroids may be associated with the development of hepatoma. ${ }^{12}$ Though cautious treatment probably carries little risk, present evidence does not indicate that adult height can be increased to a significant extent, and the advice ${ }^{13}$ that "wisdom suggests that the pediatrician stay his hand in the 'usual' case" still holds good. In the few cases in which treatment is deemed necessary because of severe emotional difficulties, testosterone, possibly given as a depot injection (testosterone oenanthate) is probably the best choice. Such treatment will lead to virilization and is contraindicated in girls. In boys it should probably be deferred until the patient is 11-12 years old and the bone age is above 9 years. ${ }^{14}$ Treatment should be started with relatively low doses and given for limited periods. Bone age must be followed closely, and, if it appears that bone maturation is outstripping growth, treatment should be reduced or stopped.

1 Smith, D. W., Fournal of Pediatrics, 1967, 70, 463

2 Bayley, N., and Pinneau, S. R., Fournal of Pediatrics, 1952, 40, 423.

Schoen, E. J., Solomon, I. L., and Milkovich, L., American fournal of Diseases of Children, 1970, 119, 252.

Sobel, E. H., Raymond, C. S., Quinn, K. V., and Talbot, N. B., Fournal of Clinical Endocrinology and Metabolism, 1956, 16, 241.

Hubble, D., and Macmillan, D. R., Archives of Disease in Childhood, 1962 37, 518.

Mellman, W. J., Bongiovanni, A. M., Garrison, M., and Steiker, D. D., Pediatrics, 1961, 28, 525

7 Geller, J., Acta Endocrinologica, 1968, 59, 307.

8 Kaplan, J. G., Moshang, T., Bernstein, R., Parks, J. S., and Bongiovanni,

A. M., Fournal of Pediatrics, 1973, 82, 38 . America, 1957, 4, 819 .

America, 1957, 4, 819.
10 Foss, G. L., Archives of Disease in Childhood, 1965, 40, 66.

11 Greene, R., Carstairs, L. S., British fournal of Clinical Practice, 1973, 27, 3.

11 Greene, R., Carstairs, L. S., British fournal of Chi

13 Bongiovanni, A. M., Pediatrics, 1961, 27, 519.

14 Bettmann, H. K., Goldman, H. S., Abramowicz, M., and Sobel, E. H., fournal of Pediatrics, $1971,79,1018$.

\section{Disorders of Lipid Metabolism}

The relationship between arterial disease, hyperlipidaemia and diet seems undoubted, though it is certainly complex. Accordingly, the "lipid profile" has arrived in many hospitals throughout the country. But physicians are uncertain about its evaluation and what treatment may or may not be indicated. While the increased demand for estimations of blood lipid reflects the clinician's increasing concern to prevent or delay the onset of arterial disease, there is still no consensus on when or how he should intervene. A symposium organized by the Association of Clinical Pathologists highlights recent advances in the knowledge of lipid metabolism and shows how fairly simple techniques can provide most of the diagnostic information required. ${ }^{1}$

We are reminded that cholesterol is not a harmful substance but an essential structural element of cell membranes, an obligatory precursor of bile acids and steroid hormones, and a constituent of plasma lipoproteins. N. B. Myant reviews its physiology and transport and discusses the mechanisms by which some agents effectively lower its concentration in the plasma.

Plasma triglycerides are also related to arterial disease, and an account of normal triglyceride metabolism provides a basis for comparison with states of abnormal lipid metabolism (D. S. Robinson). Phospholipid metabolism will be less familiar to most clinicians, and D. Gompertz focuses our attention on lecithin as the most important glycerophosphatide, both in the membrane systems and in lipidtransporting mechanisms.

Advances in analytical methods have provided the means for advancing our knowledge of lipid metabolism. The two techniques most widely used have been ultracentrifugation, followed by chemical analysis of the separated fractions, and electrophoresis. These techniques have enabled the hyperlipidaemias and hyperlipoproteinaemias to be classified, ${ }^{2}$ and this work provides the basis for the lipid profiles now emerging from our laboratories. D. G. Cramp comments on the collection of samples and on the estimation of triglycerides, cholesterol, and free fatty acids. K. Carlson (Sweden) gives detailed information on ultracentrifugation and electrophoresis, and provides useful data on the variations in triglyceride and cholesterol concentration with age and sex.

Diet influences triglyceride metabolism, and there is increasing interest in the role of triglycerides in atherosclerosis and coronary heart disease. The nature of the dietary fat accompanying carbohydrate may determine the effect of carbohydrate on the plasma triglycerides (Ian Macdonald), and this relationship is probably of greater importance in real life than the separate effect of either fat or carbohydrate. Of particular interest is the possible effect on the blood viscosity and the coagulation-fibrinolysis mechanism of the hypentriglyceridaemia induced by carbohydrate or fat.

Barry Lewis discusses the classification scheme initiated by Frederickson and colleagues ${ }^{3}$ and emphasizes that a knowledge of the plasma concentrations of cholesterol and triglyceride and of the plasma lipoprotein pattern is sufficient for the selection of treatment for most patients with hyperlipidaemia. But it is essential to realize that the accepted classification implies only that we recognize a particular lipoprotein pattern present at the time of the examination. We are classifying the plasma and not the patient, ${ }^{4}$ and there is only a limited relationship between the serum lipoprotein patterns and the underlying pathogenesis. A particular abnormal lipoprotein pattern may be caused in several different ways. At present it seems reasonable to use the World Health Organization's classification, but it represents merely a convenient shorthand for communication. As a general guide to the selection of treatment it may be somewhat complicated. In any case of hyperlipidaemia there must be a diligent search for underlying disease, and when the condition is not clearly secondary, an investigation of first degree relatives. 